\title{
Enhanced Vascular Smooth Muscle Calcium Sensitivity and Loss of Endothelial Vasodilator Influence Contribute to Myogenic Tone Development in Rat Radial Uterine Arteries during Gestation
}

\author{
Narmin Mukhtarova ${ }^{a}$ Nga Ling Ko ${ }^{a}$ Natalia I. Gokina ${ }^{a} \quad$ Maurizio Mandaláb \\ George Osola \\ ${ }^{a}$ Department of Obstetrics, Gynecology and Reproductive Sciences, University of Vermont Larner College \\ of Medicine, Burlington, VT, USA; ${ }^{b}$ Department of Biology, Ecology and Earth Science, University of Calabria, \\ Arcavacata di Rende, Italy
}

\section{Keywords}

Pregnancy - Myogenic tone - Uterine artery - Calcium .

Calcium sensitivity $\cdot$ RhoA $\cdot$ Y27632

\begin{abstract}
Uterine artery myogenic tone (MT) develops during pregnancy in hemochorial placentates such as rats and humans. The physiological reason for its appearance is not clear, and we reasoned that it may be a late pregnancy (LP) event in preparation for controlling hemorrhage during parturition. We also hypothesized that gestational increases in RhoAinduced vascular smooth muscle (VSM) calcium sensitivity are contributory and occur under the tonic influence of nitric oxide (NO). Second-order pre-placental radial arteries from early-pregnant (day 12, $n=5$ ), mid-pregnant (day 16, $n=5$ ) and LP (day 20, $n=20$ ) rats were used in combination with arteriography, VSM calcium measurements, pharmacological RHO/Rho-associated protein kinase (ROCK) and nitric oxide synthase (NOS) inhibition, and Western blotting. A subgroup of $\mathrm{LP}$ animals ( $\mathrm{LP}+\mathrm{LN} ; n=5$ ) treated with $\mathrm{L}$ NAME from gestational days 10 to 20 were used to deter-
\end{abstract}

(c) 2020 S. Karger AG, Basel

karger@karger.com

www.karger.com/jvi mine the effects of NOS inhibition on MT and RhoA expression. MT was evident throughout pregnancy, but its expression in pressurized vessels was masked by endothelial NO-induced vasodilation during early gestation. RhoA protein expression was upregulated in LP and attenuated by in vivo NOS inhibition (as was MT). In vitro RHO/ROCK inhibition decreased MT in a concentration-dependent manner without reducing VSM calcium. In summary, pressure-dependent uterine artery tone increases with gestational age due to a combination of RhoA-mediated increases in VSM calcium sensitivity and a loss of endothelial NO influence.

(c) 2020 S. Karger AG, Basel

\section{Introduction}

Pressure-dependent arterial tone is essential in blood flow regulation $[1,2]$ and for protecting smaller downstream vessels from damage due to increased blood pressure. The ambient level of tone reflects an integration of intrinsic vascular smooth muscle (VSM) contractility to pressure or stretch (myogenic tone $[\mathrm{MT}]$ ) and of other, 
superimposed vasoactive influences, for example, endothelial vasodilator (e.g., nitric oxide [NO]) release.

MT is most often present in smaller resistance arteries and has been documented in vessels from regional circulations other than the uterus, particularly from the brain and kidney [3,4]. Earlier studies in several regional circulations, including the uterine, have shown that MT results from a synergism between ionic and enzymatic mechanisms that govern VSM calcium entry and calcium sensitivity, respectively [1, 4-9].

The uterine vasculature is unique in that little or no pressure-dependent tone is present in the resistance (radial) arteries outside of pregnancy, but its extent increases considerably during gestation. This adaptation appears to be induced by local uteroplacental influences [10] and has been documented in both rats and humans $[10,11$, $21,26,27]$, although, conversely, MT is reduced in the uterine vasculature of the sheep during pregnancy $[1,11-$ 13]. This difference may be related to the type of placentation, as it is important to reduce inflow pressure in hemochorial placentas (human, rodent) to avoid villous damage, while the fetal and maternal vessels are intertwined in epitheliochorial placentas (sheep and other ungulates) in which villi are absent, and placental-inflow pressures are therefore considerably greater [14]. Thus, in view of its hemochorial placentation, the rat is a valuable animal model for understanding hemodynamic changes in human pregnancy.

We hypothesized that the appearance of MT is a late pregnancy (LP) event and that its enhancement during pregnancy arises from a combination of depolarizationinduced calcium entry and RhoA-mediated increases in VSM calcium sensitivity $[5,6]$. Based on earlier findings that showed that NO exerts an important tonic influence on RhoA expression in VSM [15-18], we also hypothesized that nitric oxide synthase (NOS) inhibition during pregnancy would reduce RhoA protein expression and that this may account for the as-yet unexplained finding that MT is nearly absent in radial arteries from pregnant rats that underwent NOS inhibition in vivo [19].

\section{Methods}

Animals, Experimental Treatments, and Blood Pressure Measurements

Twelve- to fourteen-week-old timed pregnant Sprague-Dawley $(n=30)$ rats were purchased from Charles River Laboratories (Saint-Constant, QC, Canada) and housed singly in cages at the small animal care facility at the University of Vermont, which is accredited by the American Association for Accreditation of Lab- oratory Animal Care. All animals were acclimatized for at least 4 days prior to experimentation.

The experimental design reflects the individual animal as an appropriate experimental unit for investigation. All animals were provided feed and water ad libitum under a 12D:12L photoperiod. Early-pregnant (EP, day 12/22 of gestation, $n=5$ ), mid-pregnant (MP, day $16 / 22$ of gestation, $n=5$ ), and LP (day 20/22 of gestation, $n=20$ ) rats were used in this study. Of the 20 LPs, subgroups ( $n=5$ in each) were used for NOS inhibition (in vitro), Rho-associated protein kinase (ROCK) inhibition, and Fura-2 calcium measurements (subtotal: $n=15$ ). A fourth subgroup of the LP animals $(n=5)$ was treated with $0.5 \mathrm{~g} / \mathrm{L} \mathrm{L-NAME}$ in drinking water from gestational days 10 to 20 (as in earlier studies) and designated as LP + LN [19]. Daily water intake per animal was measured on a daily basis for the latter, and blood pressures were measured noninvasively by a tail-cuff method (CODA System, Kent Scientific, Torrington, CT, USA) on the morning of each experimental day prior to euthanizing the animal for tissue collection to assure sufficient delivery of the drug and to provide a readout of its hypertensive effects. Rats were not habituated to blood pressure measurements, as they were used for confirmatory rather than comparative purposes. Five preliminary measurements were taken to relax the animal, and then an additional 10 subsequent measurements were recorded and averaged to provide a final value.

Vessels used for Western blotting were collected in addition to those used for pressure myography; hence, no additional animals were required.

Each rat was anesthetized with 3\% isoflurane, weighed, and euthanized by decapitation in a small animal guillotine. The uterus and its vasculature were removed in its entirety and pinned in a silicone-coated Petri dish filled with freshly prepared, room temperature HEPES-physiologic salt solution (PSS). All experimental protocols were approved by the Institutional Animal Care and Use Committee in accordance with NIH and ARRIVE guidelines.

\section{Isolated Vessel Preparation}

Proximal segments of second-order pre-placental radial arteries [20] were dissected free from perivascular adipose tissue. The ends of each vessel were cannulated in an arteriograph (Living Systems Instrumentation, St. Albans, VT, USA) superfused with HEPES-PSS at $37^{\circ} \mathrm{C}$, pressurized to $60 \mathrm{~mm} \mathrm{Hg}$, and equilibrated for $45 \mathrm{~min}$ under no-flow conditions. Transmural pressure and lumen diameter were recorded during each experiment using an IonOptix data acquisition program (Westwood, MA, USA).

As reported earlier [28], lumen diameter is often oscillatory in uterine arteries from pregnant rats. Hence, once the vessel response to a change in pressure stabilized, maximum and minimum values were sampled and averaged using the IonOptix software.

\section{Experimental Procedure}

Following equilibration at $60 \mathrm{~mm} \mathrm{Hg}$, intraluminal pressure in each cannulated vessel was increased to 80 and then $100 \mathrm{~mm} \mathrm{Hg}$. Each pressure step was maintained for 15-20 min to allow the vessel to reach a stable diameter. Pressure was then decreased to 60 $\mathrm{mm} \mathrm{Hg}$, and L-NAME $(100 \mu \mathrm{M})$ plus $N(\omega)$-nitro-L-arginine (LNNA, $100 \mu \mathrm{M}$ ) were added to the superfusate for $20 \mathrm{~min}$ to induce NOS inhibition prior to repeating the pressure stair.

Fully relaxed (passive) lumen diameters were obtained at the end of each experiment by superfusing each vessel for $20 \mathrm{~min}$ with a re- 
laxing solution containing $10 \mu \mathrm{M}$ diltiazem and $100 \mu \mathrm{M}$ papaverine; $n$ values represent the number of animals, with one vessel from each used for reactivity experiments, and several others collected for Western blot analyses. In the interest of clarity, $60 \mathrm{~mm} \mathrm{Hg}$, which approximates the physiologic pressure most likely experienced by uterine second-order radial arteries [14], was used in subsequent ROCK inhibition and calcium measurement experiments.

\section{Effects of p160 ROCK Inhibition with Y27632 on MT}

The effects of Y27632 were tested ( 3 and $10 \mu \mathrm{M}$ applied cumulatively; $20 \mathrm{~min}$ at each concentration) post-equilibration at $60 \mathrm{~mm}$ $\mathrm{Hg}$ (with tone) in vessels from 5 animals.

\section{Fura-2 AM Loading and Smooth Muscle Cell Cytosolic}

Calcium $\left[\mathrm{Ca}^{2+}\right]_{i}$ Measurement in Pressurized Arteries

Ratiometric measurements of Fura-2 AM fluorescence from smooth muscle cells (SMC) in the vascular wall (media) were performed using a photomultiplier system (IonOptix Inc., Milton, MA, USA). Arteries were cannulated and pressurized to $60 \mathrm{~mm} \mathrm{Hg}$. Background fluorescence was measured at $37^{\circ} \mathrm{C}$ from each artery before loading with $5 \mu \mathrm{M}$ Fura-2 AM. Ten microliters of Fura-2 AM stock solution in dehydrated DMSO was premixed with $10 \mu \mathrm{L}$ of a $20 \%$ solution of pluronic acid and added to $2 \mathrm{~mL}$ of HEPES-PSS. This solution was added to the superfusate in an arteriograph containing a radial artery pressurized to $10 \mathrm{~mm} \mathrm{Hg}$ for $60 \mathrm{~min}$ at room temperature and no circulation in the chamber to allow Fura-2 AM to penetrate into the media (VSM). After loading, arterial segments were superfused for $15 \mathrm{~min}$ at $10 \mathrm{~mm} \mathrm{Hg}$ and $37^{\circ} \mathrm{C}$ to allow intracellular de-esterification of Fura-2 AM. Background-corrected ratios of $510 \mathrm{~nm}$ emission were obtained at a sampling rate of $5 \mathrm{~Hz}$ from arteries alternately excited at 340 and $380 \mathrm{~nm}$.

Following warming to $37^{\circ} \mathrm{C}$, intraluminal pressure was increased from 10 to $60 \mathrm{~mm} \mathrm{Hg}$. The effects of Y27632 $(3,10 \mu \mathrm{M})$ on MT were tested during a 10-min exposure at each concentration. Transmural pressure, lumen diameter, and 340/380 Fura-2 ratios were recorded throughout the experimental protocol and averaged by sampling values over a period of $15 \mathrm{~s}$ (as described above for diameters). At the end of each experiment, each vessel was superfused with a relaxing solution of HEPES containing papaverine $(100 \mu \mathrm{M})+$ diltiazem $(10 \mu \mathrm{M})$ to achieve maximal relaxation as required for the calculation of tone.

\section{Western Blot Analysis}

Following uterine excision, pre-placental radial arteries from EP, LP, and LP + LN animals were collected in addition to those used for pressure myography and immediately frozen in liquid nitrogen, then kept at $-80^{\circ} \mathrm{C}$ until the day of analysis.

Arteries were lysed with Pierce RIPA buffer (ThermoFisher Scientific, Rockford, IL, USA) supplemented with Halt protease inhibitor cocktail (ThermoFisher Scientific) and homogenized in Lysis Matrix D tubes (MP Biomedicals, Solon, OH, USA) using two 30 -s pulses on a FastPrep-24 $4^{\mathrm{TM}}$ homogenizer (MP Biomedicals). Total protein concentrations were determined by BSA assay (ThermoFisher Scientific). Protein samples ( $3 \mu \mathrm{g}$ of soluble protein each) were separated using $4-15 \%$ Criterion ${ }^{\mathrm{TM}} \mathrm{TGX}^{\mathrm{TM}}$ precast gels (BioRad Laboratories, Hercules, CA, USA) and transferred to a PVDF membrane. After blocking with 5\% nonfat milk, rabbit monoclonal eNOS (1:1,000, Cell Signaling Technology, Denvers, MA, USA), rabbit polyclonal $\beta$-tubulin (1:5,000, Cell Signaling Technology), and mouse monoclonal RhoA (Cytoskeleton Inc., Denver, CO,
Table 1. Maternal body weights and arterial lumen diameters at 60 $\mathrm{mm} \mathrm{Hg}$ in relaxing solution by treatment group

\begin{tabular}{lll}
\hline Group & Body weight, $g$ & Diameter, $\mu \mathrm{m}$ \\
\hline EP & $331 \pm 10.3$ & $156 \pm 18.4$ \\
MP & $355 \pm 6.3$ & $170 \pm 39.0$ \\
LP & $416 \pm 12.5^{*}$ & $185 \pm 33.5$ \\
LP + LN & $380 \pm 13.0$ & $143 \pm 41.6$ \\
\hline
\end{tabular}

Values are expressed as mean \pm SEM; $n=5 / g$.

$* p<0.05$ versus other groups, one-way ANOVA (carried out separately for body wt and diameter data).

$\mathrm{EP}$, early pregnant; MP, mid-pregnant; LP, late pregnant; LP + $\mathrm{LN}$, late pregnant, treated with $0.5 \mathrm{~g} / \mathrm{L} \mathrm{L}-\mathrm{NAME}$ in drinking water from gestational days 10 to 20 .

USA) primary antibodies and horseradish peroxidase-conjugated secondary antibodies (Abcam, Cambridge, UK) were used to detect specific proteins on the blot. Protein bands were detected by SuperSignal West Pico Plus chemiluminescent substrate (ThermoFisher Scientific) and analyzed by densitometry using ImageJ software.

\section{Drugs and Solutions}

HEPES-PSS contained: $141.9 \mathrm{mM} \mathrm{NaCl}, 4.7 \mathrm{mM} \mathrm{KCl}, 1.7 \mathrm{mM}$ $\mathrm{MgSO}_{4}, 2.8 \mathrm{mM} \mathrm{CaCl}_{2}, 0.4 \mathrm{mM}$ EDTA, $10.0 \mathrm{mM}$ HEPES, 1.2 $\mathrm{KH}_{2} \mathrm{PO}_{4}$, and 5.0 dextrose, $\mathrm{pH}=7.4$ at $37^{\circ} \mathrm{C}$. The Fura- 2 calibration solution contained: $140 \mathrm{mM} \mathrm{KCl}, 20 \mathrm{mM} \mathrm{NaCl}, 5 \mathrm{mM}$ HEPES, $5 \mathrm{mM}$ EGTA, $1 \mathrm{mM} \mathrm{MgCl}_{2}, 5 \mu \mathrm{M}$ nigericin, and $10 \mu \mathrm{M}$ ionomycin, $\mathrm{pH}=7.4$ at $37^{\circ} \mathrm{C}$. All solutions were prepared on the day of the experiment.

Chemicals were purchased from Fisher Scientific (Fair Lawn, NJ, USA) unless stated otherwise. Ionomycin (Alfa Aesar, Ward Hill, MA, USA) and nigericin (Calbiochem, La Jolla, CA, USA) were prepared as $10 \mathrm{~mm}$ stock solutions in DMSO and kept at $-20^{\circ} \mathrm{C}$ until use. Fura-2 (Invitrogen, Carlsbad, CA, USA) was dissolved in DMSO as a $1 \mathrm{mM}$ stock solution, refrigerated in small aliquots and used within 1 week of preparation. L-NAME, papaverine, and diltiazem were purchased from Sigma-Aldrich (St. Louis, MO, USA) and L-NNA from Alfa Aesar (Haverhill, MA, USA); Y27632 (hydrochloride) was purchased from Cayman Chemical (Ann Arbor, MI, USA), dissolved in DMSO as a $10 \mu \mathrm{M}$ stock solution and frozen at $-20^{\circ} \mathrm{C}$ until use.

\section{Calculations and Statistical Analysis}

MT (\%) was calculated using the following equation: ([ $D_{\mathrm{P}}-$ $\left.\left.D_{\mathrm{A}}\right] / D_{\mathrm{P}}\right) \times 100$, where $D_{\mathrm{P}}$ is passive diameter obtained in response to papaverine and diltiazem, and $D_{\mathrm{A}}$ is active diameter of the pressurized vessels. GraphPad Prism 7.01 was used for graphical representation, calculation, and statistical analysis of data.

SMC $\left[\mathrm{Ca}^{2+}\right]_{\mathrm{i}}$ was calculated using the equation: $\left[\mathrm{Ca}^{2+}\right]_{i}=\mathrm{K}_{\mathrm{d}} \beta$ $\left(\mathrm{R}-\mathrm{R}_{\min }\right) /\left(\mathrm{R}_{\max }-\mathrm{R}\right)$, where $\mathrm{R}$ is the experimentally measured ratio $(340 / 380 \mathrm{~nm})$ of fluorescence intensity; $\mathrm{R}_{\min }$ is a ratio in the absence of $\left[\mathrm{Ca}^{2+}\right]_{i} ; \mathrm{R}_{\max }$ is a ratio at $\mathrm{Ca}^{2+}$-saturated Fura- 2 conditions, and $\beta$ is a ratio of the fluorescence intensities at $380 \mathrm{~nm} . \mathrm{R}_{\min }$, $\mathrm{R}_{\max }$, and $\beta$ were determined by an in situ calibration procedure from arteries treated with nigericin $(5 \mu \mathrm{M})$ and ionomycin $(10 \mu \mathrm{M})$ [10]. Calibration was performed on a group of vessels loaded extraluminally with Fura-2 $(n=4)$. These values were then pooled 


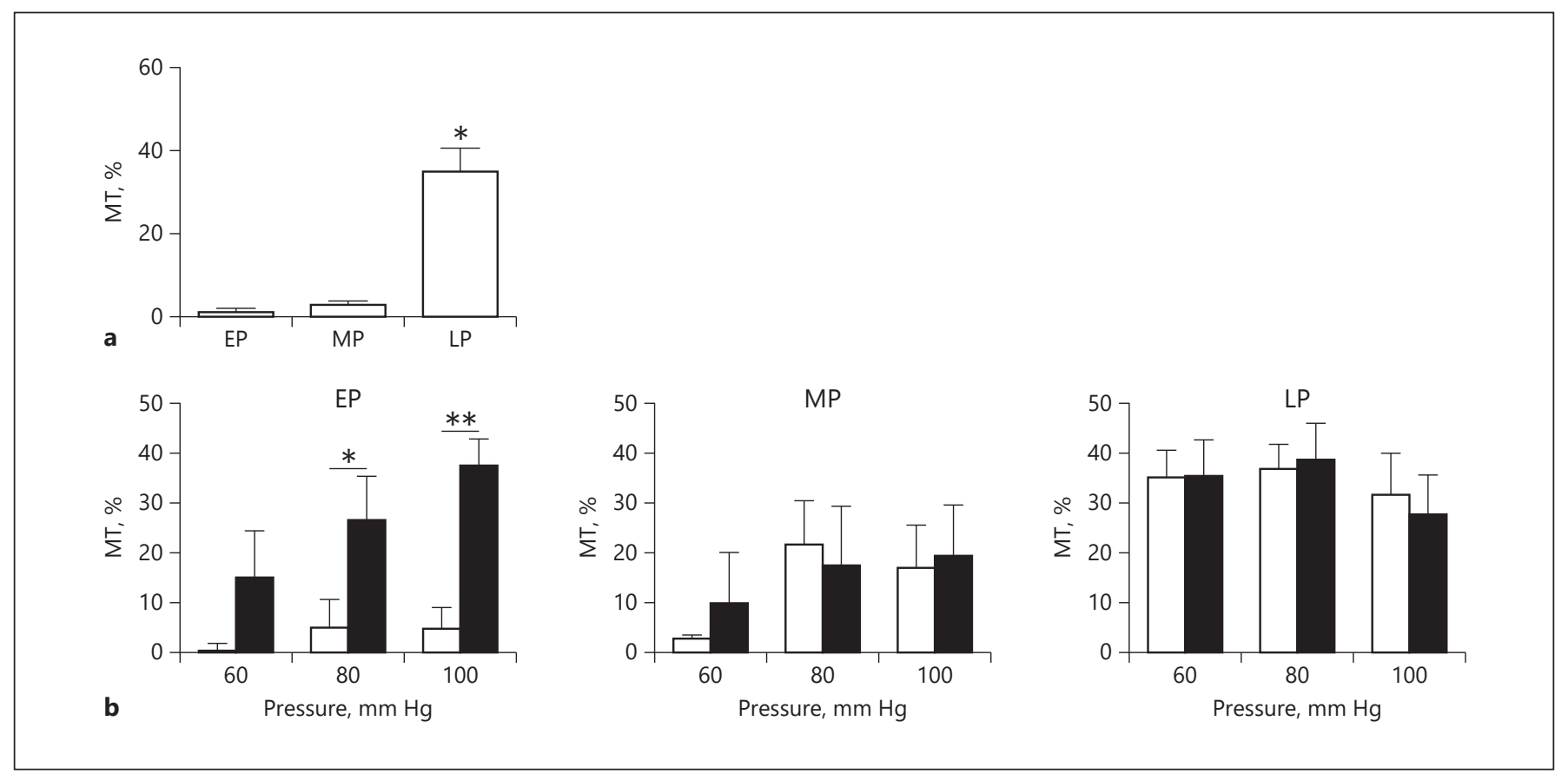

Fig. 1. a Pressure-dependent tone (\%) in isolated, pressurized (60 $\mathrm{mm} \mathrm{Hg}$ ) pre-placental second-order radial arteries from EP (day $12 / 22$ of gestation), MP (day 16/22 of gestation), and LP (day 20/22 of gestation) animals. The level of tone was significant in arteries from LP animals and essentially absent in those from the EP and MP groups ( $n=5$ /group). b Summary graph showing the level of pressure-dependent tone as a function of gestational age and transmural pressure in the absence (open bars) versus presence (shaded bars) of in vitro NOS inhibition. A significant increase in MT fol-

and used to convert the ratiometric values into actual $\left[\mathrm{Ca}^{2+}\right]_{\mathrm{i}} \cdot \mathrm{K}_{\mathrm{d}}$ (the dissociation constant for Fura-2) was $282 \mathrm{nM} \mathrm{[20].} \mathrm{Transmu-}$ ral pressure, lumen diameter, and ratio values were simultaneously recorded using an IonOptix data acquisition program and imported into SigmaPlot programs for graphical representation, calculation, and statistical analysis.

Data are expressed as mean \pm SEM. Unpaired Student $t$ test and one-way repeated measures ANOVA were used to determine the significance of differences between groups, as were non-repeated measures ANOVA (one-way and two-way, based on number of independent variables; see figure legends). Statistical differences were considered significant at $p<0.05$.

\section{Results}

\section{Animal Body Weights and Arterial Unstressed Lumen Diameters}

As shown in Table 1, LP rats were significantly heavier than the other groups. Although vessels from LP + LN rats trended smaller than those of MP or LP animals, this difference was not statistically significant. lowing NOS inhibition was only observed in vessels from EP animals at 80 and $100 \mathrm{~mm} \mathrm{Hg}$, although an effect was clearly seen at $60 \mathrm{~mm} \mathrm{Hg}$ as well. Data are expressed as means \pm SEM. * Statistical significance relative to each of the other groups, $p<0.05(n=5 /$ group, one-way ANOVA) in $\mathbf{a}$; in $\mathbf{b}$, comparisons between control and NOS-inhibited vessels were made within each gestational age and at each transmural pressure (two-way repeated measures ANOVA). ${ }^{*} p<0.05 ;{ }^{* *} p<0.01$. LP, late pregnant; MP, mid-pregnant; EP, early pregnant.

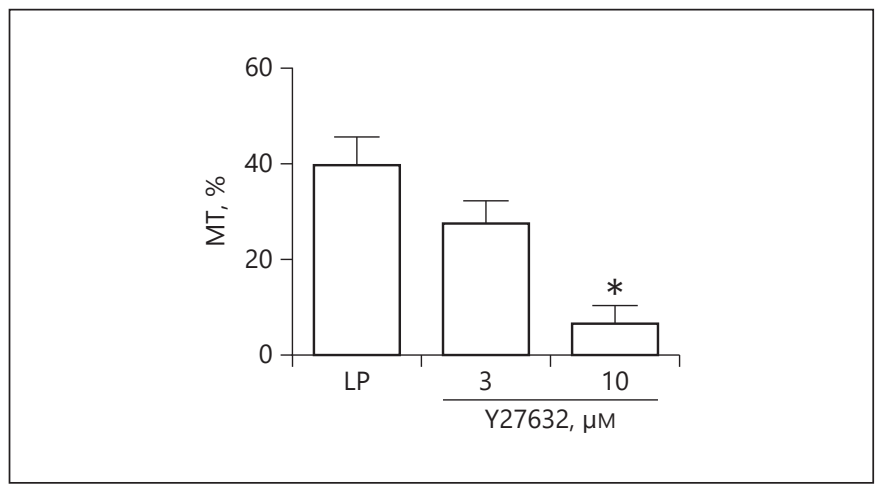

Fig. 2. Rho kinase inhibition by $10 \mu \mathrm{M}$ Y27632 significantly reduced MT in pre-placental second-order radial arteries $(60 \mathrm{~mm}$ $\mathrm{Hg}$ ) from LP animals. Some vasodilation occurred at the $3 \mu \mathrm{M}$ concentration as well, but this was not significantly different from controls. Data are expressed as means \pm SEM. ${ }^{*}$ Statistical significance relative to the other groups, $p<0.05$ ( $n=5$ /group, one-way repeated measures ANOVA). LP, late pregnant. 

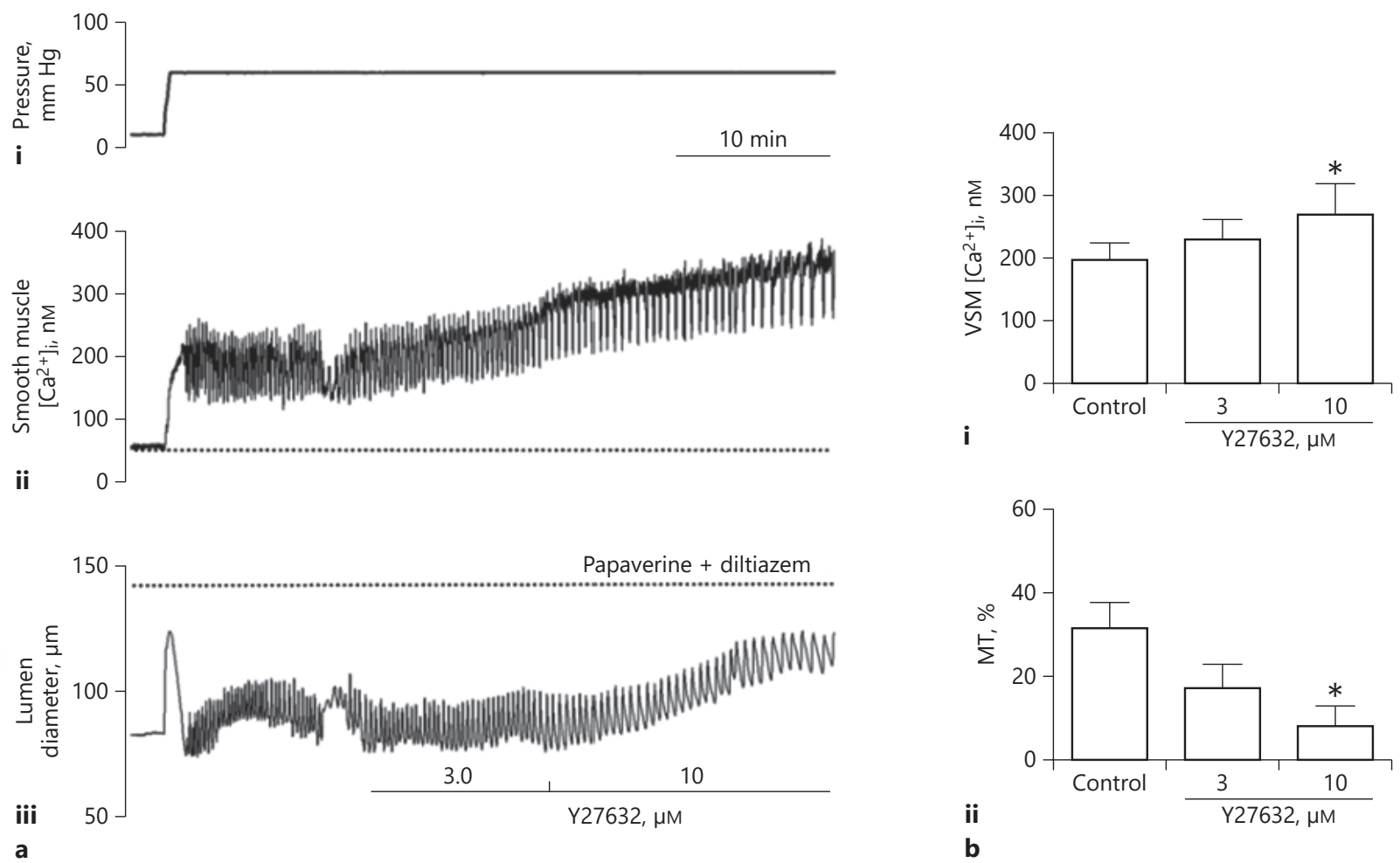

Fig. 3. Inhibition of ROCK induced a concentration-dependent increase in cytosolic $\mathrm{Ca}^{2+}$ concentration $\left[\mathrm{Ca}^{2+}\right]_{\mathrm{i}}$ and loss of MT (dilation) in pre-placental second-order radial arteries. a Representative experimental tracings showing the effects of Y27632 (3 and $10 \mu \mathrm{M})$ on VSMC cytosolic $\left[\mathrm{Ca}^{2+}\right]_{\mathrm{i}}$ and lumen diameter in one vessel from an LP rat. Note rise in calcium during the initial development of MT following the increase in intraluminal pressure from 10 to $60 \mathrm{~mm} \mathrm{Hg}$. Once vascular diameter and $\left[\mathrm{Ca}^{2+}\right]_{\mathrm{i}}$ stabilized, the addition of Y27632 produced modest increases in $\left[\mathrm{Ca}^{2+}\right]_{i}$ and loss of tone in a concentration-dependent manner. The dotted lines indicate the basal level of VSMC $\left[\mathrm{Ca}^{2+}\right]_{\mathrm{i}}$ (ii trace) and the re-

\section{Time Course and Extent of MT Development during} Rat Pregnancy; Effects of NOS Inhibition

Pressure-dependent tone at $60 \mathrm{~mm} \mathrm{Hg}$ was minimal in arteries from EP $(1.5 \pm 0.52 \%)$ and MP $(3.1 \pm 0.57 \%)$ animals but considerable $(35 \pm 5.4 \%)$ in LP vessels $(n=5 /$ group, $p<0.05$; Fig. 1a). As shown in Figure 1b, tone was somewhat increased at higher pressures $(80$ and $100 \mathrm{~mm}$ $\mathrm{Hg}$ ) and NOS inhibition (L-NAME + L-NNA added to the superfusate) significantly enhanced pressure-dependent tone in EP (at 80 and $100 \mathrm{~mm} \mathrm{Hg}$ ) but not in MP or LP vessels. laxed lumen diameter (iii trace) of the same artery in the presence of $100 \mu \mathrm{M}$ papaverine and $10 \mu \mathrm{M}$ diltiazem. As noted earlier [28], vasomotion is characteristic of uterine arteries from pregnant rats. b Summary graphs showing VSMC cytosolic $\left[\mathrm{Ca}^{2+}\right]_{\mathrm{i}}$ increase (i), and the significant decrease in \% MT (ii) in response to $10 \mu \mathrm{M}$ Y27632 in pre-placental second-order radial arteries pressurized to $60 \mathrm{~mm} \mathrm{Hg}$. The effects of $3 \mu \mathrm{M}$ Y 27632 were intermediate in both cases. Data are expressed as means \pm SEM. * Statistical significance, $p<0.05$ vs. control ( $n=5$ /group, one-way repeated measures ANOVA). VSM, vascular smooth muscle; MT, myogenic tone.

\section{Effects of ROCK Inhibition on MT and VSM Cytosolic} Calcium

The application of $10 \mu \mathrm{M} Y 27632$, a potent inhibitor of p160 ROCK [21], to vessels from LP rats reduced the extent of MT by $85 \pm 7.0 \%(p<0.05)$, with intermediate vasodilation evident in response to the $3 \mu \mathrm{M}$ concentration as well (Fig. 2).

This loss of tone was not associated with any decreases in vascular SMC cytosolic $\left[\mathrm{Ca}^{2+}\right]_{\mathrm{i}}$. Rather, as shown in the tracing (Fig. 3a), and in the data summary (Fig. 3b), $\left[\mathrm{Ca}^{2+}\right]_{\mathrm{i}}$ increased progressively in response to each con- 


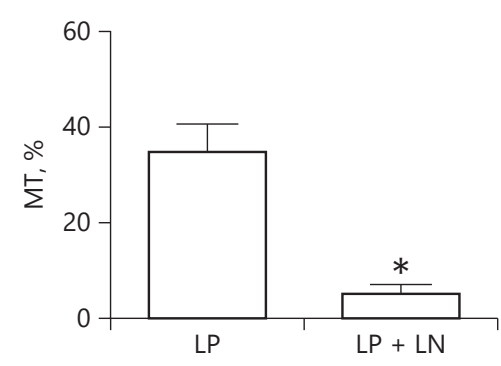

Fig. 4. MT was significantly ( $>80 \%)$ reduced in pressurized preplacental second-order radial arteries from NOS-inhibited (LNAME, $0.5 \mathrm{~g} / \mathrm{L}$ from days 10 to 20 of gestation) versus control LP rats. Data are expressed as means \pm SEM. * Statistical significance, $p<0.05$ relative to LP control $(n=5 /$ group, unpaired Student $t$ test). MT, myogenic tone.

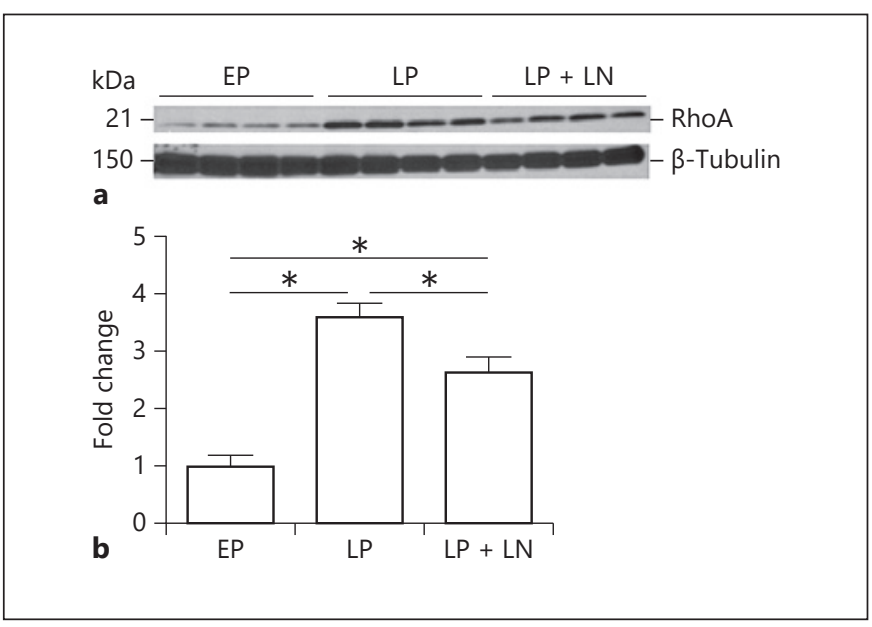

Fig. 5. Comparison of RhoA protein expression in pre-placental second-order radial arteries from EP (day 12/22 of gestation), LP (day 20/22 of gestation), and LP L-NAME-treated (LP + LN) rats. a Western blot gel showing RhoA and $\beta$-tubulin protein expression in vessels from EP, LP, and LP + LN rats. b Densitometric analysis normalized to $\beta$-tubulin. Compared to arteries from LP rats, RhoA protein expression was significantly lower in vessels from EP and LP + LN groups. Data are expressed as means $\pm \mathrm{SE}$. * Statistical significance, $p<0.05$ ( $n=4$ /group, one-way ANOVA). $\mathrm{EP}$, early pregnant; LP, late pregnant.

centration of Y27632, significantly so at the higher (10 $\mu \mathrm{M})$ concentration.

\section{Effects of in vivo NOS Inhibition on Mean Arterial \\ Blood Pressures and MT}

Mean arterial pressures at the end of the 10-day treatment with L-NAME $(0.5 \mathrm{~g} / \mathrm{L})$ averaged $119 \pm 6.6 \mathrm{~mm} \mathrm{Hg}$ $(n=5)$ in LP $+\mathrm{LN}$ animals, some $20 \mathrm{~mm} \mathrm{Hg}$ higher than that mean arterial pressures normally measured in control animals [19].

In vivo NOS inhibition with L-NAME from gestational days 10 to 20 prevented the development of significant MT in radial arteries from LP animals $(n=5$ /group, $p<$ 0.05; Fig. 4); the level of tone in LP-LN vessels was reduced by $>80 \%$ compared with controls.

\section{RhoA Protein Expression in Vessels from EP and $L P$}

Control and from LP NOS-Inhibited $(L P+L N)$ Rats

RhoA protein was detectable in vessels from EP animals, and its expression was significantly increased in vessels from LP animals (Fig. 5). RhoA protein also increased significantly in radial arteries from rats that underwent NOS inhibition with L-NAME in vivo (from days 10 to 20 of pregnancy), but to a level that was between that of vessels from EP and LP animals, and significantly different from both (Fig. 5).

\section{Discussion}

Effects of Gestational Age and Transmural Pressure on Pressure-Dependent versus Myogenic Tone

The physiological purpose underlying the appearance of uterine artery pressure-induced tone in LP is not known but may have to do with increasing flow resistance in order to prevent excessive downstream (spiral artery) pressures which could otherwise lead to accelerated placental inflow and fetal villous damage [22] and/or possibly limiting hemorrhage during parturition.

As shown in Figure 1, pressure-dependent tone at 60 $\mathrm{mm} \mathrm{Hg}$ was minimal in vessels from EP and MP animals and significant in those from LP rats. At higher pressures ( 80 and $100 \mathrm{~mm} \mathrm{Hg}$, Fig. 2), pressure-dependent tone was increased somewhat in arteries from EP and MP animals as well, although the values were significantly lower than those of arteries from LP animals.

Interestingly, the in vitro NOS inhibition data in Figure $1 \mathrm{~b}$ show that significant MT is present in EP as well but is almost completely inhibited by the vasodilatory influence of endothelial NO, since NOS inhibition increased tone at every pressure and significantly so at 80 and $100 \mathrm{~mm} \mathrm{Hg}$. This overriding effect of NO was no longer present by MP. Thus, the capacity of VSM to develop MT does not appear to be a LP phenomenon; rather, VSM is capable of myogenic responsiveness throughout pregnancy, but its manifestation earlier in pregnancy is masked by the overriding effect of endothelial NO. This 
influence is reduced and eventually lost as pregnancy progresses such that considerable MT is present in vessels from LP rats, and the effects of NOS inhibition become insignificant.

\section{Species Differences in MT during Pregnancy}

With the exception of one report in mice [23], most published studies on rodents, rabbits, and humans have documented increases in uterine artery MT during pregnancy $[19,20,24-28]$. This is quite different from the situation in the ewe, in which uterine artery MT during gestation is decreased. One explanation for the species differences may relate to the type of placentation. Ungulates such as sheep have epitheliochorial placentas, in which the maternal vascular network is preserved, and intertwines with the fetal placental vessels so that the exchange of nutrients and wastes occurs from vessel to vessel. Accordingly, intravascular pressures in the small maternal arteries are reportedly close to systemic in epitheliochorial placentates (84 mm Hg [14]), whereas in species with hemochorial placentation (such as humans, rats, and guinea pigs), they are on the order of only 9-14 $\mathrm{mm} \mathrm{Hg}$ [14], since the placenta essentially forms a chamber that is perfused with maternal blood in a high-volume, low-velocity flow pattern. Since blood pressures in the main uterine arteries are close to systemic [14], a considerable pressure drop occurs along the length of the radial arteries, and the presence of MT thus provides an effective mechanism for regulating flow resistance in a bidirectional manner.

\section{Physiological Mechanisms Responsible for Altering \\ Uterine Artery MT during Gestation}

Our experimental results are consistent with previously published studies in the uterine and other regional circulations (e.g., cerebral) showing that pressure-induced MT arises from a combination of ionic and enzymatic mechanisms that regulate cytosolic calcium and calcium sensitivity, respectively. As shown in the uterine, cerebral, and renal circulations, pressure-induced VSM depolarization leads to calcium entry through L-type voltage-dependent calcium channels [29]. The contractile effect of increased cytosolic calcium is further augmented by the activation of calcium-sensitizing enzymes such as protein kinase $\mathrm{C}$ and/or RhoA which alter the balance between myosin light chain kinase and phosphatase activity in a way that favors actomyosin interaction [4, 30-32].

For example, Zhang and colleagues have described several vascular adaptations that underlie the gestational reduction in MT in uterine arteries from the ewe. These include (1) upregulation of large conductance calcium- activated potassium $\left(\mathrm{BK}_{\mathrm{Ca}}\right)$ channel activity [11], (2) increased calcium sparks and spontaneous outward currents that activate $\left(\mathrm{BK}_{\mathrm{Ca}}\right)$ channels and elicit VSM hyperpolarization [11], (3) downregulation of actin polymerization [12], and (4) a reduction in calcium sensitivity due to an attenuation of protein kinase C/extracellular signalregulated kinase signaling $[18,33]$. Many of these changes can be mimicked in nonpregnant animals by chronic treatment of nonpregnant tissues with 17 beta-estradiol and progesterone, underscoring the role of sex steroids in their genesis.

As already discussed, in rats, rabbits, and humans, the consensus is that pressure-induced MT is increased in pregnancy $[19,20,24-28]$ and that both calcium handling and calcium sensitivity mechanisms are augmented. For example, both decreased function (rather than expression) of voltage-gated potassium $\left(\mathrm{K}_{\mathrm{v}}\right)$ channels [28] and upregulation of VSM RhoA signaling as a means for increasing VSM calcium sensitivity (this study) have been observed.

One report in mice [23] described a reduction in MT in the main uterine artery and attributed it to a combination of increased endothelial NO release and gap junctional communication. In our experience, there is no tone in the main uterine artery of the rat (data not shown), but there are functional differences based on arterial location $[10,20]$; clearly, additional studies are needed to clarify differences in arterial tone based on vascular architecture and species.

In terms of the molecular mechanisms underlying uterine artery tone development during pregnancy, less is known about rodents than sheep. Using a model of unilateral oviductal ligation (which results in a "half-pregnant" rat since implantation occurs in only one uterine horn), we found that MT was only present in vessels in the implanted horn, suggesting that local uteroplacental influences may be responsible [10]. If, as in sheep, sex steroids are involved (especially progesterone, since estrogen is produced in the ovaries and not the placenta of a pregnant rat $[34,35])$, this raises the question of why the effect is localized to only the implanted horn, since arterial steroidal concentrations are increased systemically and should therefore equally affect vessels in both uterine horns.

One as-yet putative pathway is venoarterial communication, a process in which placental signals secreted into the uterine vein pass across the venous wall into the periarterial mesometrial space to modulate arterial structure and tone. We recently found that redirecting the venous outflow by removing a segment of vein next to an artery significantly reduced the expansive (outward) remodeling in the adjacent arterial segment [36]. This is the first 
in vivo evidence implicating venoarterial communication in the process of maternal uterine arterial remodeling. It also raises the possibility that the process of arterial expansive remodeling itself may alter VSM function, for example, MT may be induced through changes in gene expression that occur during the remodeling process.

\section{Mechanisms Underlying Upregulation of RhoA Signaling}

This is the first study to show that it is RhoA signaling is largely responsible for MT in the rat radial artery of LP rats, since its inhibition by incubation with Y27632, a selective p160ROCK inhibitor, reduced MT by $>80 \%$. Although the mechanisms through which RhoA is upregulated during pregnancy in the maternal uterine circulation are unknown, several studies by Sauzeau et al. [15-17] deserve mention because they showed that RhoA expression in VSM is tonically regulated by endothelial NO.

Specifically, in vivo treatment with L-NAME significantly reduced RhoA signaling in rat aortas and pulmonary arteries, and this effect was prevented by oral co-administration of sildenafil $[15,16]$. In rat or human arterial SMCs, sodium nitroprusside or 8-(2-chlorophenylthio)cGMP induced a rise in RhoA mRNA and protein expression, and this could be inhibited by (R[p])-8-bromo-betaphenyl-1,N(2)-ethenoguanosine $3^{\prime}: 5^{\prime}$-phosphorothioate, a PKG inhibitor. In a subsequent study [17], treatment with the phosphodiesterase-5 inhibitor sildenafil restored and augmented RhoA, substantiating VSM cGMP-PKG pathway as the responsible pathway. These effects of NOcGMP signaling were linked to both stimulation of RhoA gene transcription and a post-transcriptional increase in RhoA protein stability.

With respect to our results on the effects of gestation, this is quite plausible since NO signaling is augmented in pregnancy on a systemic level [37], and locally in the uterine circulation, as first shown by the 8-fold increases in calcium-dependent NOS activity (and expression) in uterine arteries from pregnant versus nonpregnant women $[38,39]$. Many subsequent studies by Magness and Bird (reviewed in [40] and [41]) and others, including our own $[14,37]$, have confirmed the importance of NO signaling in mediating gestational changes in uterine artery reactivity and remodeling.

Here, in vivo NOS inhibition with L-NAME from days 10 to 20 of pregnancy significantly reduced RhoA protein expression within the uterine artery wall and reduced the level of arterial tone by $>80 \%$, as in earlier studies $[19,20]$. The RhoA effect was intermediate, that is, based on the Western blotting intensity analysis, RhoA expression ap-

Uterine Artery MT during Pregnancy proximately tripled in vessels from LP control animals, but only doubled in rats treated with L-NAME $(0.5 \mathrm{~g} / \mathrm{L}$, added to drinking water).

\section{Limitations and Considerations}

The use of isolated, pressurized vessels eliminates a number of factors extrinsic to the vascular wall that may impact on the extent of arterial tone present in vivo, such as humoral/hormonal or neural influences, and physical forces such as pulsatile intravascular pressure and the shear stress of blood flowing over the endothelial luminal surface.

Likewise, although endothelial NO is probably the major influence on uterine artery VSM, L-NAME inhibition is not specific to NOS3 (eNOS), and it is therefore not possible to attribute the changes in MT and in RhoA expression specifically to an altered influence of endothelial NO. Many other cell types generate NO and these may, in turn, affect the arterial wall via direct paracrine action (e.g., macrophages, nerves, or mast cells) or indirectly (e.g., through changes in intravascular pressure). Although we inhibited NO signaling, endothelial denudation was not included in the experimental design, and some endothelial influence from other endothelium-derived vasoactive molecules cannot be excluded.

Although NOS inhibition has been used as an animal model for human preeclampsia, like most animal models, it only approximates human disease. It would be interesting to examine uterine artery MT in another animal model of preeclampsia (such as sFlt-1 or STOX1 overexpression) or, better yet, in human intra-myometrial vessels obtained from normal versus preeclamptic women during cesarean section, as in earlier studies [27, 42].

We also do not know the extent to which L-NAME inhibited the gestational upregulation of NO signaling in the uterine circulation. Measuring this parameter in vivo accurately is difficult since blood or urinary measurements reflect systemic and not local (uterine) changes, and a number of factors that modulate NOS activation (e.g., estrogen, endothelial cytosolic calcium, tetrahydrobiopterin, oxidative state, etc.) are both interactive and altered during mammalian gestation.

In summary, the findings of this study delineate the extent and basis of uterine resistance (radial) artery pressure-dependent MT as a function of gestational age and show that increases in Rho-A signaling may contribute to the manifestation of tone by increasing VSM calcium sensitivity. They also indicate that intrinsic MT is obscured by NO during early gestation and that this influence decreases with gestational age, resulting in augmented va- 
soconstriction. The physiologic utility of tone increasing late in pregnancy is not known, and we speculate that it may contribute to the regulation of uteroplacental blood flow as gestation progresses and/or with limiting hemorrhage during parturition. Clearly, evaluation of this property under in vivo conditions would be useful in providing context into its physiologic relevance.

\section{Acknowledgment}

The authors would like to acknowledge the support of the NIH National Heart and Lung Institute (NHLBI) in carrying out this study.

\section{Statement of Ethics}

As stated in the text, all experimental protocols and procedures were approved by the Institutional Animal Care and Use Committee in accordance with NIH and ARRIVE guidelines.

\section{Disclosure Statement}

The authors have no conflicts of interest to declare.

\section{Funding Sources}

NIHLBI RO1 HL134371 (GO).

\section{Author Contributions}

The concept and experimental design were conceived by G.O., M.M., and N.L.K. N.I.G. and N.M. carried out the Fura-2 AM calcium measurement experiments and N.M. carried out the reactivity experiments and, with the help of N.L.K., the Western blots. N.L.K. and N.M. analyzed and graphed the data and wrote the draft. G.O. managed the study and edited the final draft, which was then proofed by all the authors.

\section{References}

1 Davis MJ, Hill MA. Signaling mechanisms underlying the vascular myogenic response. Physiol Rev. 1999 Apr;79(2):387-423.

2 Halpern W, Osol G. Influence of transmural pressure of myogenic responses of isolated cerebral arteries of the rat. Ann Biomed Eng. 1985;13(3-4):287-93.

3 Loutzenhiser R, Griffin K, Williamson G, Bidani A. Renal autoregulation: new perspectives regarding the protective and regulatory roles of the underlying mechanisms. Am J Physiol Regul Integr Comp Physiol. 2006 May;290(5):R1153-67.

4 Gokina NI, Park KM, McElroy-Yaggy K, Osol G. Effects of Rho kinase inhibition on cerebral artery myogenic tone and reactivity. J Appl Physiol (1985). 2005 May;98(5):1940-8.

5 Johnson RP, El-Yazbi AF, Takeya K, Walsh EJ, Walsh MP, Cole WC. Ca2+ sensitization via phosphorylation of myosin phosphatase targeting subunit at threonine- 855 by Rho kinase contributes to the arterial myogenic response. J Physiol. 2009 Jun;587(Pt 11):253753.

6 Gokina NI, Knot HJ, Nelson MT, Osol G. Increased $\mathrm{Ca} 2+$ sensitivity as a key mechanism of PKC-induced constriction in pressurized cerebral arteries. Am J Physiol. 1999 Sep; 277(3):H1178-88.

7 Geary GG, Osol GJ, Longo LD. Development affects in vitro vascular tone and calcium sensitivity in ovine cerebral arteries. J Physiol. 2004 Aug;558(Pt 3):883-96.

8 Osol G. Mechanotransduction by vascular smooth muscle. J Vasc Res. 1995 Sep-Oct; 32(5):275-92.
9 Gokina NI, Osol G. Temperature and protein kinase $\mathrm{C}$ modulate myofilament $\mathrm{Ca} 2+$ sensitivity in pressurized rat cerebral arteries. Am J Physiol. 1998 Jun;274(6):H1920-7.

10 Gokina NI, Kuzina OY, Fuller R, Osol G. Local uteroplacental influences are responsible for the induction of uterine artery myogenic tone during rat pregnancy. Reprod Sci. 2009 Nov;16(11):1072-81.

$11 \mathrm{Hu}$ XQ, Song R, Romero M, Dasgupta C Huang X, Holguin MA, et al. Pregnancy Increases Ca2+ Sparks/Spontaneous Transient Outward Currents and Reduces Uterine Arterial Myogenic Tone. Hypertension. 2019 Mar; 73(3):691-702.

12 Xiao D, Huang X, Yang S, Longo LD, Zhang L. Pregnancy downregulates actin polymerization and pressure-dependent myogenic tone in ovine uterine arteries. Hypertension. 2010 Nov;56(5):1009-15.

13 Hu XQ, Xiao D, Zhu R, Huang X, Yang S, Wilson $\mathrm{S}$, et al. Pregnancy upregulates large-conductance $\mathrm{Ca}(2+)$-activated $\mathrm{K}(+)$ channel activity and attenuates myogenic tone in uterine arteries. Hypertension. 2011 Dec;58(6):1132-9.

14 Moll W, Künzel W. The blood pressure in arteries entering the placentae of guinea pigs, rats, rabbits, and sheep. Pflugers Arch. 1973 Jan;338(2):125-31.

15 Sauzeau V, Le Jeune H, Cario-Toumaniantz C, Smolenski A, Lohmann SM, Bertoglio J, et al. Cyclic GMP-dependent protein kinase signaling pathway inhibits RhoA-induced $\mathrm{Ca} 2+$ sensitization of contraction in vascular smooth muscle. J Biol Chem. $2000 \mathrm{Jul}$ 275(28):21722-9.
16 Sauzeau V, Rolli-Derkinderen M, Marionneau C, Loirand G, Pacaud P. RhoA expression is controlled by nitric oxide through cGMP-dependent protein kinase activation. J Biol Chem. 2003 Mar;278(11):9472-80.

17 Sauzeau V, Rolli-Derkinderen M, Lehoux S, Loirand G, Pacaud P. Sildenafil prevents change in RhoA expression induced by chronic hypoxia in rat pulmonary artery. Circ Res. 2003 Oct;93(7):630-7.

18 Xiao D, Buchholz JN, Zhang L. Pregnancy attenuates uterine artery pressure-dependent vascular tone: role of PKC/ERK pathway. Am J Physiol Heart Circ Physiol. 2006 Jun; 290(6):H2337-43.

19 Barron C, Mandala M, Osol G. Effects of pregnancy, hypertension and nitric oxide inhibition on rat uterine artery myogenic reactivity. J Vasc Res. 2010;47(6):463-71.

20 Gokina NI, Mandalà M, Osol G. Induction of localized differences in rat uterine radial artery behavior and structure during gestation. Am J Obstet Gynecol. 2003 Nov;189(5):148993.

21 Ishizaki T, Uehata M, Tamechika I, Keel J, Nonomura K, Maekawa M, et al. Pharmacological properties of Y-27632, a specific inhibitor of rho-associated kinases. Mol Pharmacol. 2000 May;57(5):976-83.

22 Burton GJ, Woods AW, Jauniaux E, Kingdom JC. Rheological and physiological consequences of conversion of the maternal spiral arteries for uteroplacental blood flow during human pregnancy. Placenta. 2009 Jun;30(6): $473-82$. 
23 Veerareddy S, Cooke CL, Baker PN, Davidge ST. Vascular adaptations to pregnancy in mice: effects on myogenic tone. Am J Physiol Heart Circ Physiol. 2002 Dec;283(6):H222633.

24 Eckman DM, Gupta R, Rosenfeld CR, Morgan TM, Charles SM, Mertz H, et al. Pregnancy increases myometrial artery myogenic tone via NOS- or COX-independent mechanisms. Am J Physiol Regul Integr Comp Physiol. 2012 Aug;303(4):R368-75.

25 Osol G, Cipolla M. Interaction of myogenic and adrenergic mechanisms in isolated, pressurized uterine radial arteries from late-pregnant and nonpregnant rats. Am J Obstet Gynecol. 1993 Feb;168(2):697-705.

26 Cipolla MJ, Binder ND, Osol G. Myoendometrial versus placental uterine arteries: structural, mechanical, and functional differences in late-pregnant rabbits. Am J Obstet Gynecol. 1997 Jul;177(1):215-21.

27 Kublickiene KR, Cockell AP, Nisell H, Poston $\mathrm{L}$. Role of nitric oxide in the regulation of vascular tone in pressurized and perfused resistance myometrial arteries from term pregnant women. Am J Obstet Gynecol. 1997 Nov 177(5):1263-9.

28 Telezhkin V, Goecks T, Bonev AD, Osol G, Gokina NI. Decreased function of voltagegated potassium channels contributes to augmented myogenic tone of uterine arteries in late pregnancy. Am J Physiol Heart Circ Physiol. 2008 Jan;294(1):H272-84.
29 Ledoux J, Werner ME, Brayden JE, Nelson MT. Calcium-activated potassium channels and the regulation of vascular tone. Physiology (Bethesda). 2006 Feb;21(1):69-78.

30 Morgan KG, Leinweber BD. PKC-dependent signalling mechanisms in differentiated smooth muscle. Acta Physiol Scand. 1998 Dec;164(4):495-505.

31 Horowitz A, Menice CB, Laporte R, Morgan KG. Mechanisms of smooth muscle contraction. Physiol Rev. 1996 Oct;76(4):967-1003.

32 Hill MA, Falcone JC, Meininger GA. Evidence for protein kinase $\mathrm{C}$ involvement in arteriolar myogenic reactivity. Am J Physiol. 1990 Nov;259(5 Pt 2):H1586-94.

33 Xiao D, Huang X, Yang S, Zhang L. Direct chronic effect of steroid hormones in attenuating uterine arterial myogenic tone: role of protein kinase c/extracellular signal-regulated kinase 1/2. Hypertension. 2009 Aug;54(2): 352-8.

34 Matt DW, MacDonald GJ. In vitro progesterone and testosterone production by the rat placenta during pregnancy. Endocrinology. 1984 Aug;115(2):741-7.

35 Al-Bader MD. Estrogen receptors alpha and beta in rat placenta: detection by RT-PCR, real time PCR and Western blotting. Reprod Biol Endocrinol. 2006 Mar;4(1):13.
36 Ko NL, Mandalà M, John L, Gelinne A, Osol G. Venoarterial communication mediates arterial wall shear stress-induced maternal uterine vascular remodeling during pregnancy. Am J Physiol Heart Circ Physiol. 2018 Sep; 315(3):H709-17.

37 Osol G, Ko NL, Mandalà M. Altered Endothelial Nitric Oxide Signaling as a Paradigm for Maternal Vascular Maladaptation in Preeclampsia. Curr Hypertens Rep. 2017 Sep; 19(10):82.

38 Nelson SH, Steinsland OS, Suresh MS, Lee NM. Pregnancy augments nitric oxide-dependent dilator response to acetylcholine in the human uterine artery. Hum Reprod. 1998 May;13(5):1361-7.

39 Nelson SH, Steinsland OS, Wang Y, Yallampalli C, Dong YL, Sanchez JM. Increased nitric oxide synthase activity and expression in the human uterine artery during pregnancy. Circ Res. 2000 Sep;87(5):406-11.

40 Bird IM, Zhang L, Magness RR. Possible mechanisms underlying pregnancy-induced changes in uterine artery endothelial function. Am J Physiol Regul Integr Comp Physiol. 2003 Feb;284(2):R245-58.

41 Boeldt DS, Bird IM. Vascular adaptation in pregnancy and endothelial dysfunction in preeclampsia. J Endocrinol. 2017 Jan; 232(1):R27-44.

42 Terp GE, Christensen IT, Jørgensen FS. Structural differences of matrix metalloproteinases. Homology modeling and energy minimization of enzyme-substrate complexes. J Biomol Struct Dyn. 2000 Jun;17(6):933-46. 